\title{
A UWB Power Control Algorithm Based on Activity Link
}

\section{Protection}

\author{
Xuefeng Pan ${ }^{\mathrm{a}}$,Jing Hu, Jiaheng Cao \\ Department of Computer Science, Luojia College,Wuhan University, 430064 Wuhan, China
}

\begin{abstract}
In the wireless local area network, the mobile terminal by a large carrier power consumption, which greatly influence the performance of mobile, in order to reduce the energy consumption of a control algorithm to establish the link. During the link establishment, the power controlled multiple access mode, using a modified maximum tolerate interference based on the (MEI) channel access algorithm for distributed power control. The simulation results show that, with the increasing distance, the link between the multiuser interference decreases, can effectively improve the efficiency of wireless local area network.
\end{abstract}

Key words:ultra wide band(UWB), wireless sensor network(WSN), match-filter(MF), energy-detection(ED)

\section{Introduction}

The technology of traditional radio is based on the carrier, but on the modulation signals to the carrier, the modulation is carried out on the sender and the receiver must carry on the demodulation. It increases the complexity of hardware, and larger power consumption is needed to launch a carrier, the mobile terminals mobile performance caused great influence. Under this background, the federal communications commission (FCC) definition of civil $\mathrm{UWB}^{[1]}$ (the technology of Ultra wide band). The technology of UWB has the characteristics of low power consumption, high bandwidth and low complexity $^{[2-3]}$, and the communication technology of traditional wireless has many different places. UWB is particularly suitable for indoor and other places of dense multipath high-speed wireless access.

At present, in the research of wireless sensor network, strengthen the signal and reduce energy consumption and so on all make for correlation. Literature 3 by using the algorithm to enhance the signal coverage, literature 4 from the perspective of reducing energy consumption to improve the relevant agreements. In the link level control energy consumption by power control algorithm, can effective improve the efficiency of the network.

\section{Analysis of UWB Link}

\subsection{Analysis of UWB signal}

UWB signal is defined as the signal bandwidth is more than $500 \mathrm{MHz}$, the ratio of the signal bandwidth and center frequency between $1 \% \sim 25 \%$ for broadband, less than $1 \%$ for narrowband, visible all the bandwidth of the UWB significantly greater than the current technology of communication bandwidth ${ }^{[4-5]}$.

Relative bandwidth of signal is defined as: the relative bandwidth $=\left(\mathrm{f}_{\mathrm{H}}-\mathrm{f}_{\mathrm{L}}\right) /\left[\left(\mathrm{f}_{\mathrm{H}}+\mathrm{f}_{\mathrm{L}}\right) / 2\right]^{[6]}$. If relative bandwidth is greater than 0.25 , it is a super wideband signal. Uwb baseband transmission, realizing the way is to send impulse radio signal transmit voice and image data, can send up to 1 billion per second on behalf of the pulse signal of $0 \mathrm{~s}$ and $1 \mathrm{~s}$. The pulse signal frequency domain is very wide, range in a few $\mathrm{Hz}$ to several $\mathrm{GHz}$, the low frequency part can realize communications through walls. Because the transmission power of the signal is very low, only the equivalent of some background noise, not to interfere with other narrowband signal ${ }^{[7]}$.Because only when needed to send out the pulse wave, thus greatly reducing the power consumption.

\subsection{Analysis of UWB Modulation Method}

UWB transmission power of restricted by the transmit power spectral density, and thus affect the choice of modulation mode in two ways. One is for every bit of energy modulation will need to provide the best error performance. The second is the choice of modulation scheme affect the structure of the signal power spectral density, therefore may add some additional restrictions on the transmission power.

There are three kinds of UWB modulation mode: direct sequence spread spectrum UWB (DS - UWB), jump in ultra wideband (TH - UWB) and multiband UWB. Because binary reverse polarity code has better properties than the PPM code, design the TH - PAM modulation, namely jump add binary code when reverse polarity 
modulation.Under the same ber requirements, binary reverse polarity signal transmission power is only half the binary orthogonal PPM modulation.

In this kind of modulation, time is divided into frames. $\mathrm{T}_{\mathrm{f}}$ is time of each frame, and each frame is composed of $\mathrm{N}_{\mathrm{h}}$. It tentative $\mathrm{N}_{\mathrm{h}}=7 . \mathrm{T}_{\mathrm{c}}$ is the time of each time slot, so $\mathrm{T}_{\mathrm{f}}=\mathrm{T}_{\mathrm{c}} * \mathrm{~N}_{\mathrm{h}}$. A bit of information with $\mathrm{N}_{\mathrm{s}}$ pulse, said here $\mathrm{N}_{\mathrm{s}}$ $=4$. A user within A frame to send only one pulse, so the user A's A bit of information in the above four frames after the $\mathrm{T}_{\mathrm{f}}$ sent to receive completely.

Assume that the $\mathrm{TH}$ code of user $\mathrm{A}$ is $\{0,1,5,3\}$, therefore, user $\mathrm{A}$ in the first frame 0 time slot detection, in order to distinguish between A bit of information, here using the binary reverse polarity PAM modulation. Here the first frame detected pulse to -1.By the same token, the receiver in the first time slot in the second frame, the third time slot 5 frames, the fourth frame timeslot distinguish 3 detection, the signal respectively $+1,+1$ and -1 . At this point, A user of A bit of information represented by $A$ pulse test is completed, all for the $\{-1,+1,+1,-1\}$.

According to the characteristics of uwb signal, assuming there are $\mathrm{N}$ users in the system, so the link $\mathrm{i}$ the signal-to-noise ratio of formula is as follows:

$$
\gamma_{i}=\frac{P_{i} g_{i i}}{R_{i}\left(\eta_{i}+\sigma^{2} T_{f} \sum_{j=1, j \neq i}^{N} P_{j} g_{i j}\right)}
$$

Among them, $\mathrm{N}$ is the number of links. $\mathrm{T}_{\mathrm{f}}$ is each time frame. $R_{i}$ is the link rate of bits of information,and $g_{i j}$ is link gain from the sending node $\mathrm{j}$ to the receiving node $\mathrm{i} . \eta_{\mathrm{i}}$ is receiving nodes of the ith link I background noise power spectral density. $\sigma^{2}$ is the formation of the UWB pulse factor.

A bit by NS pulse, $\mathrm{T}_{\mathrm{f}}$ is a frame of time, such the time of a bit completely sent is $\mathrm{T}_{\mathrm{b}}=\mathrm{T}_{\mathrm{f}} * \mathrm{~N}_{\mathrm{s}}$. In this way, the bit rate $\mathrm{Ri}=1 / \mathrm{Tb}$, average power $\mathrm{P}_{\mathrm{i}}=\mathrm{E} / \mathrm{t}=\mathrm{E}_{\text {frame }} / \mathrm{T}_{\text {frame }}=$ $\mathrm{E}_{\mathrm{m}, \mathrm{i}} / \mathrm{T}_{\text {frame }}=\mathrm{E}_{\mathrm{m}, \mathrm{i}} / \mathrm{T}_{\mathrm{f}}=\mathrm{E}_{\mathrm{m}, \mathrm{i}} / \mathrm{T}_{\mathrm{f}}$, among them, the $\mathrm{E}_{\text {frame }}$ is the power of link $i$ to send in one frame. $E_{m, i}$ is the energy of a pulse.Therefore, in order to change the power of link i, it can by changing the pulse energy or changing the $T_{f}$. The changes of $\mathrm{T}_{\mathrm{f}}$ can be divided into $\mathrm{Nh}$ and $\mathrm{Tc}$.

\section{The Algorithm of Power Control}

\subsection{The Main Idea}

In establishing a link, multiple access method adopts an improved distributed power control based on MEI channel access algorithm.Network topology in the form of the AD - hoc network, each link between don't need to be synchronized, only need to synchronization between receiver and send points.For the upper bound of the transmission power, as UWB spectrum are unauthorized frequency band, if transmitted power of UWB without control, is likely to cause interference to other systems. So you have to consider the upper bound of the transmission power, therefore using FCC rules here.

\subsection{Establish Linkment}

In order to facilitate described the algorithm of power control, first introduced the concept of largest can tolerate interference (MEI).The biggest can tolerate interference is a link in order to keep normal communication QoS and is able to bear the biggest additional interference.If link $\mathrm{i}$ target the minimum signal-to-noise ratio is $\gamma_{\mathrm{i}}^{\mathrm{T}}$, ultra-wideband signal-to-noise ratio according to the formula, the calculation formula of MEI below:

$$
\gamma_{i}^{T}=\frac{P_{i} g_{i i}}{R_{i}\left(\eta_{i}+\sigma^{2} T_{f} \sum_{j=1, j \neq i}^{N} P_{j} g_{i j}+\sigma^{2} T_{f} M_{i}\right)}
$$

Each link, regularly check the interference around me, and his own gamma information such as $\gamma_{i}{ }^{T}$ and $R_{i}$.It can work out their own Mi by formula 2 .

When a new link to be created, assuming that the system has $\mathrm{N}$ activities links in the link, each link has been worked out their own Mi, and timing will send these information through radio channel. When another new link to build, it first listening out of all the links of $\mathrm{Mi}, 1 \leqslant \mathrm{i} \leqslant \mathrm{N}$. Suppose $\mathrm{N}+1$ the link rate of signal to noise ratio and requirements are: $\gamma_{\mathrm{N}+1}^{\mathrm{T}}$ and $\mathrm{R}_{\mathrm{N}+1}$.

$$
\gamma_{N+1}^{T}=\frac{P_{N+1} g_{N+1, N+1}}{R_{N+1}\left(\eta_{N+1}+\sigma^{2} T_{f} \sum_{j=1}^{N} P_{j} g_{N+1, j}\right)}
$$

In order to guarantee the minimum rate $\mathrm{R}_{\mathrm{i}}$ to send, the power requirements is:

$$
P_{N+1}=\frac{\gamma_{N+1}^{T} R_{N+1}\left(\eta_{N+1}+\sigma^{2} T_{f} \sum_{j=1}^{N} P_{j} g_{N+1, j}\right)}{g_{N+1, N+1}}
$$

Already exists in the system $\mathrm{N}$ the activities of the link, the link priority is higher than the new link. When $\mathrm{N}+1$ the link be established, must make the pledge that we now have normal communication link, so the article $\mathrm{N}+1$ link first calculate $\min \left(\mathrm{M}_{\mathrm{i}} / \mathrm{g}_{\mathrm{i}, \mathrm{N}+1}\right), 1 \leqslant \mathrm{i} \leqslant \mathrm{N}$. And considering the FCC masking power, it is concluded that the upper bound of the power of the $\mathrm{N}+1$ link limit: $\mathrm{P}_{\max }=$ $\min \left(\mathrm{P}_{\mathrm{FCC}}, \min \left(\mathrm{M}_{\mathrm{i}} / \mathrm{g}_{\mathrm{i}, \mathrm{N}+1}\right)\right), 1 \leqslant \mathrm{i} \leqslant \mathrm{N}$.

Compared with $\mathrm{P}_{\mathrm{N}+1}$ Pmax values, if $\mathrm{P}_{\mathrm{N}+1} \leq \mathrm{P}_{\max }$, can be successfully established the new link; Otherwise unable to build new links, has reached the awaited state.After a random experiment to try to access it again.So if after a time out later still cannot be established, the $\mathrm{N}+1$ link will be the issue to top processing, or reduce the rate of demand. When the $\mathrm{P}_{\mathrm{N}+1}=\mathrm{P}_{\max }$, clearly link the $\mathrm{N}+1$ to send Pmax. When the $\mathrm{P}_{\mathrm{N}+1} \leq \mathrm{P}_{\max }$, the best is sending power $\mathrm{P}_{\mathrm{opt}} \in\left\{\mathrm{P}_{\mathrm{N}+1}, \mathrm{P}_{\max }\right\}$.In reference [1] in order to calculate the Popt, MEI algorithm designed a kind of balance, but the algorithm is too complex.For the sake of simplicity, we adopted a compromise, take $\mathrm{P}_{\mathrm{opt}}=\left(\mathrm{P}_{\mathrm{N}+1}+\mathrm{P}_{\mathrm{max}}\right) / 2$. 


\subsection{Algorithm Description}

\subsubsection{The Sending Node Algorithm Description}

(1)Initialize a link establishment request, and send the request information to the $\mathrm{RX}$ node.

(2)Calculate the node in the case of without interfering with the existing other link, can use one of the biggest power $\mathrm{P}_{\max }$.

(3)When it receive the reply the RX node minimal power requirements $\mathrm{P}_{\min }$, to (4), otherwise wait.

(4)Compare, if $\mathrm{P}_{\min } \leqslant \mathrm{P}_{\max }$, the link can be successfully established, turn to (5), or the link cannot be established, after a random time, return to(1) the initialization step.

(5)Calculate the optimal power $P_{\text {opt }}$, successfully established and sent to link status information to RX node.

(6)Start sending data.

\subsubsection{The Receiving Node Algorithm Description}

(1)Check its receive buffer, if received the TX initialization link request information, then transferred to (2), otherwise return to (1).

(2)Calculate the oneself in order to maintain the required $\mathrm{SNR}, \mathrm{P}_{\min }$, and sent to the TX.

(3)When you receive the success to establish a link information, the TX to (4), otherwise it return to (1).

(4)Began to receive data.

\subsection{Algorithm Implementation}

UWB power control algorithm based on activity link protection according to the sending and receiving nodes of two parts. Algorithm implementation program includes two main parts: the first part is a custom message class powerMsg, definition to carry power control information. The second part is sent and received the core code of the algorithm.

\subsubsection{The Definition of Class PowerMsg}

Custom powerMsg classes, the main tag carries information about power control, including size, link power gain, SNR, node status information (free or busy) and so on.

message powerMsg

\{

fields:

double txPower;

double rxWantMinpower;

double MEI;

int sourceNodeIndex;

int destintNodeIndex;

int $\mathrm{x}$;

int $y$;

bool wantCommWithYou=false;

bool linkSucessToCreat $=$ false;

\}

After this MSG files by compiling, can generate the other two files: powerMsg_m.h and powerMsg_m.cc.

\subsubsection{The Core Code of the Sending and Receiving Node Information}

Create a module in the physical.Main processing module and the physical channel information, such as the calculation of the signal-to-noise ratio of the two nodes, compute a total interference, the receiving node calculation are receiving node MEI, calculates the maximum send power sending node, and so on.

(1)Calculate the signal-to-noise ratio of the two nodes double Physic::getGain(powerMsg *pmsg)

\{

double gain;

double dist=getDistance(pmsg);

if (dist $<35$ )

$\{$ gain $=1$;

\}

gain=1225/(dist*dist);

return gain;

\}

(2)Calculation of total interference a receive node double Physic::getInterference()

\{

double totleNiose $=0.0$;

for (int $\mathrm{i}=0 ; \mathrm{i}<\operatorname{txpmsg}$ Array.items ()$; \mathrm{i}++)$

\{

powerMsg *pmsg=(powerMsg *)txpmsgArray[i];

if $(($ pmsg-> getSourceNodeIndex ()$) !=($ parentModule () $->\operatorname{index}()))$

\{

double gains $=$ getGain $(\mathrm{pmsg})$;

totleNiose $+=($ pmsg- $>$ getTxPower ()$) *$ gains;

\}

\}

return totleNiose;

\}

(3)Calculate the MEI is receiving node

double Physic::getmyMEI(double tSIR)

\{

double interfere $=$ getInterference () ;

double xixi=tSIR*requireRate*shapingFactor*Tf; double

myMei=(initxPower*myLinkGain-tSIR*requireRate*

backnoise-xixi*interfere) /(xixi);

return myMei;

(4) Calculate the maximum send power sending node double Physic::maxPower()

\{

double thetxPower;

double maxpower $=1000.0$; for (int $\mathrm{i}=0 ; \mathrm{i}<\operatorname{rxpmsgArray.items}() ; \mathrm{i}++)$ \{

powerMsg *pmsgs=(powerMsg *)rxpmsgArray[i]; double gain=getGain (pmsgs);

if $((($ pmsgs- $>$ getMEI ()$) /$ gain $)<$ maxpower $)$

maxpower $=($ pmsgs- $>$ getMEI ()$) /$ gain; 


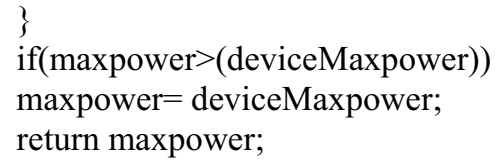

\section{The Simulation Experiment and Result Analysis}

For UWB simulation USES is OMNeT++, it is open source component based modular open network simulation platform $^{[9]}$.

When the power control algorithm simulation, the TH PAM modulation, the comprehensive binary reverse polarity jump code modulation. Binary reverse polarity required by the code under the same bit error rate, a signal transmitted power is only half the binary orthogonal PPM modulation. Test information in TH - PAM modulation modulation, time is divided into individual frames, each frame of $\mathrm{T}_{\mathrm{f}}$, expressed in four pulse one bit of information. A user within a frame to send only a pulse. Test information of one bit of information in four frames after the $\mathrm{T}_{\mathrm{f}}$ sent to receive completely. Through the system after the adjustment information is converted into the corresponding binary sequence and pulse form users to send.

Will be set up to send information, combined algorithm simulation program, through the node link signal strength and after into the algorithm to optimize signal spectrum ratio on the simulation analysis of signal source.

Establish a node link simulation diagram is shown in figure 1 , the blue point said mobile terminal, black has told the arrow link.

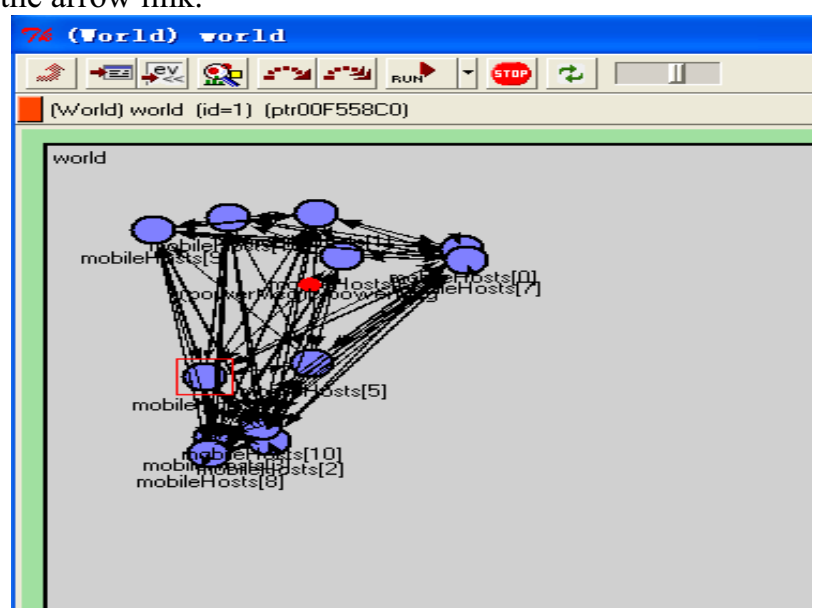

Figure 1. the Link of Node.

Set the signal frequency is $3.389 \mathrm{GHz}$, rate of 100 MHZ UWB signal.Figure 2 and figure 3 is before and after the use of power control algorithm for UWB signal contrast test figure, can see the power of UWB narrow pulse signal increased to 6.18 from $-8.56 \mathrm{dBm} \mathrm{dBm}$, gain of $14.74 \mathrm{dBm}$.Considering the actual test of the line loss, about 2-3 DBM.The actual gain 11 DBM, effectively enhance the quality of UWB signal.

Through the above simulation experiment, it can be seen that using UWB as the physical carrier, multi-user interference between each link is very small.It is very suitable for application in more than one user of the system, and complex multipath environment, so as to meet the needs of UWB short high-speed data transmission system.

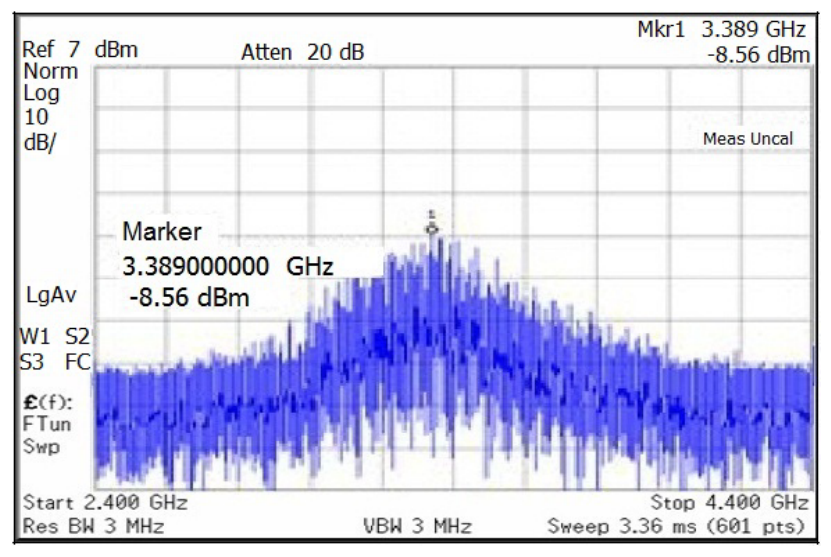

Figure 2. the Spectrum of Source Signal.

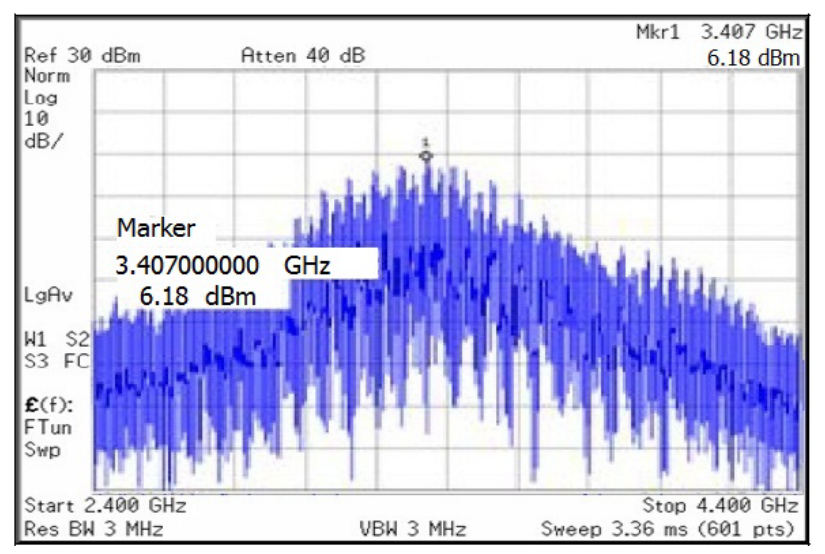

Figure 3. Signal Spectrum after Using Power Control Algorithm

\section{Conclusion}

For mobile terminal for launch carrier consumed power and reduce the mobility of the larger problem, this paper proposes a control algorithm to build links.In establishing a link, the power control multiple access methods, for distributed power control based on MEI channel access algorithm is improved.After the improvement, reduce the energy consumption and the link between the multi-user interference is very small, the multiple users of wireless sensor network (WSN) life cycle.

\section{Acknowledgment}

The scientific research project of Education Department of Hubei Province under Grant No.B2015291. The education science planning project of Hubei Province under Grant No.2015GB156. 


\section{References}

1. Ding Rui, Qian Zhi-hong, Wang Xue. UWB positioning system based on joint TOA and DOA estimation[J]. Journal of Electronics \& Information Technology, 2010, 32(2): 313-317.

2. Muruganathan S D, Ma D C F, Bhasin R I, etal. A centralized energy-efficient routing protocol for wireless sensor networks. IEEE Communications Magazine, 2005, 43(3):8-13.

3. Zhang Hua, Song Zheng-xun, Shi Yun, et al. Research of TOA ranging technology based on UWB[J]. Journal of Jilin University (Information Science Edition), 2008, 26(1): 106- 110.

4. Tang Yong,Zhou Mingtian,Zhang Xin.Routing protocol for wireless sensor networks research progress[J]. Journal Of Software,2006,17 (3):410-421.

5. LIU Zhi-jun, LI Na-yuan, YANG Shao-hua. Base on energy balance LEACH-DC protocol design[J]. Computer engineering \& design, 2012, 33(4): 13371341.

6. Shen Bo,Zhang Shiyong.wireless sensor network clustering routing protocol[J].Journal Of Software, 2006, 17(7): 1588- 1600 .

7. LI Chun-xia,ZHAN Yong-zhao,WANG Liang-min. Secure Localization Scheme for Wireless Sensor Networks Based on UWB and Mobile Anchors[J]. Journal of Chinese Computer Systems,2012,33(2):347- 352. 